\title{
Correction to: Frugals, Militants and the Oil Market
}

Etienne Billette de Villemeur and Pierre-Olivier Pineau

\section{Correction to:}

Chapter 15 in: P.-O. Pineau et al. (eds.),

Games in Management Science, International Series in

Operations Research \& Management Science 280, https://doi.org/10.1007/978-3-030-19107-8_15

The chapter was published with an error in splitting the first name and surname of the author Etienne Billette de Villemeur online. The same has now been corrected in all versions of the book.

Also, the Table of Contents has been updated in the front matter. 